\title{
Engineering of New UV-Blocking Hollow Proteinoid Nanoparticles of Narrow Size Distribution Containing All-trans Retinoic Acid for Biomedical Applications
}

Belostozky A, Kolitz-Domb M, Grinberg I, Haham H and Margel S*

Department of Chemistry, The Institute of Nanotechnology and Advanced Materials, Bar Ilan University, Ramat Gan, Israel

\begin{abstract}
All-trans retinoic acid (at-RA), the most active form of vitamin A, is known to be highly beneficial in dermatology. At-RA can reduce acne vulgaris symptoms and improve the skin appearance significantly. Moreover, at-RA is a useful treatment for different skin diseases and for several types of cancer. However, it is extremely sensitive when exposed to ultraviolet (UV) light, due to conjugated double bonds that comprise its chemical structure. In order to increase the benefits of topical use of at-RA, a new drug carrier encapsulating and protecting at-RA from light-dependent degradation, is designed and presented here. Proteinoids are biocompatible polymers made from amino acids by thermal step-growth polymerization. These polymers form hollow nanoparticles in an aqueous solution by a simple selfassembly process, during which suitable molecules may be encapsulated within the particles. Thus, newly designed UV-absorbing proteinoids were utilized to encapsulate at-RA acid. New proteinoids were synthesized by thermal stepgrowth polymerization of glutamic acid, phenyl alanine and tyrosine in absence or presence of the UV absorber paraaminobenzoic acid. The proteinoids were of relatively high molecular weights and narrow molecular weight distributions (42-84 kDa, PDls of 1.02-1.12). At-RA, was then successfully encapsulated (up to $20 \%$ ) within the self-assembled proteinoid nanoparticles dispersed in an aqueous continuous phase. The proteinoid nanoparticles were able to protect the at-RA from light dependent degradation up to $94 \%$ over $24 \mathrm{~h}$, while under similar conditions free at-RA degraded entirely over $3 \mathrm{~h}$. The study also indicates that both the hollow and retinoic acid-filled particles are non-toxic and cellpermeable in $\mathrm{HaCaT}$ cells, a human epithelial cell line. The study suggests that at-RA-filled proteinoid nanoparticles protect at-RA from light-dependent degradation, offering significant advantage over free at-RA. Therefore, the optimal proteinoid particles chosen may potentially be used for acne vulgaris treatment as well as other biomedical applications requiring UV-protected retinoic acid.
\end{abstract}

Keywords: Proteinoid nanoparticles; All-trans retinoic acid; Acne vulgaris; UV-protection; Para-amino benzoic acid

\section{Introduction}

In recent years, all-trans retinoic acid (at-RA, Figure 1) and its derivatives (retinoids), both natural and synthetic, have been popular additives in cosmetics. At-RA is recognized as a gold standard for the prevention and treatment of several dermatologic disorders, such as acne vulgaris and photo-aged skin [1,2]. Acne vulgaris is a very common dermatologic disorder, with more than $80 \%$ of the population suffering from it during their lives $[3,4]$. Acne vulgaris is known as a multifactorial disease with various symptoms, including comedones, nodules, papules, cysts and pilosebaceous inflammation. Topical administration of at-RA can increase the turnover of follicular epithelial cells and facilitate comedolysis, thereby reducing acne symptoms and improving the skin appearance $[5,6]$. Moreover, at-RA can benefit the skin elasticity and moisture and mitigate fine wrinkles by increasing the production of collagen and elastin $[7,8]$. Furthermore, at-RA is known to be beneficial for many auto-immune skin disease, skin cancer as well as being a chemotherapeutic drug for other types of cancer [9-12]. At-RA functions by entering the cell and the cell nucleus and activating the retinoic acid receptors (RAR and RXR), thus regulating the transcription of different sets of genes [13].

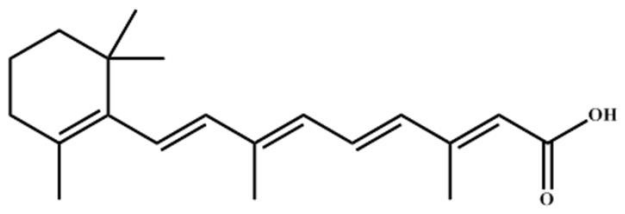

Figure 1: Chemical structure of at-retinoic acid
Yet, at-RA is extremely sensitive to ultraviolet (UV) light (280-400 $\mathrm{nm}$ ), due to the presence of conjugated double bonds [14]. Hence, when topically applied and exposed to any light source, at-RA is unstable and demonstrates extensive conversion to less potent forms [14].

Hereafter, new delivery systems can help in overcoming such drawbacks of topical at-RA treatment. Encapsulation of at-RA in suitable delivery systems such as biodegradable nanoparticles (NPs) can provide protection against UV-light, allowing increased amount of at-RA to reach the skin. Furthermore, different types of NPs are known to be skin permeable, due to their small size and spherical shape, while not detected by the immune system $[15,16]$. Therefore, NPs can be used as appropriate drug carriers to treat different skin conditions.

Proteinoids are random polymers comprised of amino acids and synthesized by thermal step-growth polymerization [17-22]. These polymers have many advantages, proteinoids are biodegradable, non-toxic and non-immunogenic due to their resemblance to natural proteins $[23,24]$. One important advantage is that proteinoids can be

*Corresponding author: Shlomo Margel, Department of Chemistry, The Institute of Nanotechnology and Advanced Materials, Bar Ilan University, Ramat Gan 52900 Israel, Fax: 97235318791; Tel: 97235318861; E-mail: shlomo.margel@biu.ac.il

Received: September 26, 2017; Accepted: October 03, 2017; Published: October 10, 2017

Citation: Belostozky A, Kolitz-Domb M, Grinberg I, Haham H, Margel S (2017) Engineering of New UV-Blocking Hollow Proteinoid Nanoparticles of Narrow Size Distribution Containing All-trans Retinoic Acid for Biomedical Applications. J Nanomed Nanotechnol 8: 462. doi: 10.4172/2157-7439.1000462

Copyright: (c) 2017 Belostozky A, et al. This is an open-access article distributed under the terms of the Creative Commons Attribution License, which permits unrestricted use, distribution, and reproduction in any medium, provided the original author and source are credited. 
Citation: Belostozky A, Kolitz-Domb M, Grinberg I, Haham H, Margel S (2017) Engineering of New UV-Blocking Hollow Proteinoid Nanoparticles of Narrow Size Distribution Containing All-trans Retinoic Acid for Biomedical Applications. J Nanomed Nanotechnol 8: 462. doi: 10.4172/21577439.1000462

Page 2 of 9

designed according to their desired use, by choosing the appropriate amino acid combination for their particular function. This design feature can provide a major leverage for proteinoids in biomedical applications including drug delivery systems.

After the thermal step-growth polymerization, proteinoids can fold in an aqueous solution, through a self-assembly mechanism, to form hollow particles. The self-assembly process of the proteinoid particles occurs due to the many functional groups which are part of the random polymer backbone. When proteinoids are self-assembled in suitable conditions, the hydrophobic residues form a hydrophobic core in the inner side of the particle, in order to minimize their contact with water, while the hydrophilic groups remain on the outer side of the particle and form hydrogen bonds with the surrounding water [25,26]. This self-assembly process may be utilized to encapsulate different suitable molecules within the core of the particles, achieving a biocompatible drug carrier [27]. In recent studies, our group used this procedure to encapsulate anti-cancer drugs and near infrared fluorescent dyes within nano-sized particles for cancer therapy and detection [24,28,29].

In the present study, a series of proteinoids was designed in order to form NPs that protect at-RA from UV-light.

\section{Materials and Methods}

\section{Materials}

The following analytical-grade chemicals were purchased from commercial sources and were used without further purification: L-glutamic acid (Glu), L-tyrosine (Tyr), L-phenylalanine (Phe), paraaminobenzoic acid (PABA), all-trans retinoic acid (at-RA), sodium hydroxide $(\mathrm{NaOH})$, super pure HPLC water, super pure HPLC acetonitrile, super pure HPLC trifluoroacetic acid, sodium chloride $(\mathrm{NaCl})$, dimethyl sulfoxide (DMSO), trehalose, phosphate buffered saline (PBS), Cyanine3 (Cy3), Cyanine7 (Cy7), N-hydroxysuccinimide (NHS) ester and Hoechst stain were all purchased in $\geq 98 \%$ purity from Sigma, Israel. Water was purified by passing deionized water through an Elgastat Spectrum reverse osmosis system from Elga Ltd. (High Wycombe, UK). Dialysis membranes (1 kDa), Dulbecco's modification of Eagle's medium (DMEM), fetal bovine serum (FBS), glutamine, penicillin/streptomycin and mycoplasma detection kit were purchased from Biological Industries (Bet Haemek, Israel). Cell cytotoxicity lactate dehydrogenase (LDH) assay kit was purchased from BioVision (USA). Alexa Fluor ${ }^{\circledR} 680$ wheat germ agglutinin (WGA) was purchased from Invitrogen (USA). HaCaT cell line was kindly provided by Prof. Eli Sprecher from the dermatology department of Tel Aviv Sourasky Medical Center, Israel.

\section{Synthesis of proteinoids by thermal step-growth polymerization}

A mixture of amino acids and PABA ( $5 \mathrm{~g}$ ) was heated to a molten state by a heat mantle under $\mathrm{N}_{2}$ atmosphere at $180^{\circ} \mathrm{C}$, and stirred by a mechanical stirrer at $150 \mathrm{rpm}$ for $45 \mathrm{~min}$. The product is a highly viscous orange-brown paste, which hardens to give a glassy mass upon cooling to room temperature. After cooling, the residue was extracted by $20 \mathrm{ml}$ of $0.15 \mathrm{M} \mathrm{NaOH}$ solution and lyophilized to yield the solid proteinoid material [30]. This process was repeated with different ratios (weight\%) of PABA, yielding Prot. 1-5 as described in Table 1.

\section{Proteinoid characterization}

The molecular weights and polydispersity index of the dried crude proteinoids were determined at $70^{\circ} \mathrm{C}$ using GPC consisting of a Waters Spectra Series P100 isocratic HPLC pump with an ERMA ERC-7510

\begin{tabular}{|l|c|c|c|}
\hline Proteinoid & $\boldsymbol{\alpha}$-Amino acids content & $\begin{array}{c}\boldsymbol{\alpha} \text {-Amino acids } \\
\text { ratio (w/w/w) }\end{array}$ & $\begin{array}{c}\text { PABA percentage } \\
\text { (weight\%) }\end{array}$ \\
\hline Prot. 1 & Glu, Phe, Tyr & $1: 1: 1$ & 0 \\
\hline Prot. 2 & Glu, Phe, Tyr & $1: 1: 1$ & 1 \\
\hline Prot. 3 & Glu, Phe, Tyr & $1: 1: 1$ & 5 \\
\hline Prot. 4 & Glu, Phe, Tyr & $1: 1: 1$ & 10 \\
\hline Prot. 5 & Glu, Phe, Tyr & $1: 1: 1$ & 20 \\
\hline
\end{tabular}

Prot. 1-5 were prepared as described in the methods section. Weight of the total monomers content is $5 \mathrm{~g}$.

Table 1: Composition of the different proteinoids.

refractive index detector and a Rheodyne (Coatati, CA) injection valve with a $20 \mu \mathrm{l}$ loop (Waters, MA). The samples were eluted with super pure HPLC water through a linear BioSep SEC-s3000 column (Phenomenex) at a flow rate of $1 \mathrm{ml} / \mathrm{min}$. The molecular weights of the proteinoids were determined relative to poly(ethylene glycol) standards (Polymer Standards Service-USA, Silver Spring, MD) with a molecular weight range of $100-450000 \mathrm{Da}$ and the natural proteins human serum albumin $(67 \mathrm{kDa})$ and bovine plasma fibrinogen $(340$ $\mathrm{kDa}$ ), using Clarity chromatography software.

The optical activities of the proteinoids were determined using a PE 343 polarimeter (PerkinElmer). All measurements were performed in water $(1 \mathrm{mg} / \mathrm{ml})$ at $589 \mathrm{~nm}$ and $25^{\circ} \mathrm{C}$.

The absorption spectra of the proteinoids were obtained by using a Cary 100 UV-visible spectrophotometer (Agilent Technologies Inc.). The measurements were performed in $0.1 \mathrm{~N} \mathrm{NaOH}$ aqueous solution $(1 \mathrm{mg} / \mathrm{ml})$ at $25^{\circ} \mathrm{C}$, using a Cary Eclipse spectrophotometer (Agilent Technologies Inc.).

\section{Proteinoid particle preparation}

Proteinoid NPs were prepared by a self-assembly mechanism. Briefly, $10 \mathrm{mg}$ of the dried proteinoid were added to $10 \mathrm{ml} 10^{-5} \mathrm{M} \mathrm{NaCl}$ aqueous solution. The mixture was then heated to $80^{\circ} \mathrm{C}$ and stirred at $250 \mathrm{rpm}$, until the crude proteinoid dissolved completely. Due to the poor solubility of at-RA in water, it was first dissolved in DMSO and then added to the proteinoid mixture, prior to the particle formation (0.5-1\% of the particles aqueous dispersion). For this purpose, an appropriate concentration of at-RA powder $(1-30 \% \mathrm{w} / \mathrm{w}$ relative to the proteinoid) was weighed and dissolved in DMSO and heated to $80^{\circ} \mathrm{C}$. After both solutions reached $80^{\circ} \mathrm{C}$, the at-RA solution was added to the proteinoid mixture. The mixture was left to cool to room temperature, in order to form the at-RA-filled proteinoid NPs. After preparation, the at-RA-filled proteinoid NPs aqueous dispersion was dialyzed extensively through a cellulose dialysis membrane (1000 Da MWCO) against distilled water to remove the DMSO. Then, the particle dispersion was filtered through a $3 \mu \mathrm{m}$ glass microfiber membrane syringe filter (VWR EU, England) to remove excess at-RA crystals. Hollow proteinoid NPs were prepared in the same manner, excluding the at-RA.

\section{Size and size distribution}

Hydrodynamic diameter and size distribution of the NP aqueous dispersion was measured at room temperature with a particle DLS analyzer Vasco 2 Particle Sizing System (Cordouan Technologies SAS, France).

The size and size distribution of a few of the NPs were also measured with a cryogenic transmission electron microscope (cryoTEM). For this purpose, a small droplet of an aqueous dispersion of the particles was placed on a perforated lasy carbon film supported 
Citation: Belostozky A, Kolitz-Domb M, Grinberg I, Haham H, Margel S (2017) Engineering of New UV-Blocking Hollow Proteinoid Nanoparticles of Narrow Size Distribution Containing All-trans Retinoic Acid for Biomedical Applications. J Nanomed Nanotechnol 8: 462. doi: 10.4172/21577439.1000462

Page 3 of 9

on a TEM copper grid held by tweezers. The drop was blotted with a piece of filter paper, resulting in the formation of thin films of 100 $300 \mathrm{~nm}$. The specimen was subsequently plunged into a reservoir of liquid ethane cooled by liquid nitrogen to ensure its vitrification (rapid freezing) and to prevent ice crystal formation. The vitrified specimen was transferred under liquid nitrogen and mounted on a cryogenic sample holder cooled to $-170^{\circ} \mathrm{C}$. All samples were observed under lowdose conditions. Vitrified samples were examined in an FEI T12 G2 Cryo-TEM operating at $120 \mathrm{kV}$ and equipped with a Gatan 626 cryoholder system. The mean diameter was determined by measuring at least 200 particles using image analysis software (AnalySIS Auto, Soft Imaging System GmbH, Germany).

\section{$\zeta$-Potential}

The particles surface potential was measured in aqueous dispersion in $\mathrm{pH}=7$ and at a concentration of $1 \mathrm{mg} / \mathrm{ml}$, using $\zeta$-potential analyzer, Zetasizer 3000 HSa model (Malvern Instruments Company, England).

\section{HPLC}

HPLC analysis was carried out by Spectra System HPLC equipped with a UV/vis detector (Thermo Scientific, USA) and a reverse phase C18 column $(75 \mathrm{~mm} \times 4.6 \mathrm{~mm}$, Phenomenex, USA). The mobile phase was water and acetonitrile, both containing $0.1 \%$ aqueous solution of trifluoroacetic acid at a flow rate of $1 \mathrm{ml} / \mathrm{min}$, wavelength was set at $350 \mathrm{~nm}$ [31]. Calibration standard solutions was prepared and used by diluting an appropriate volume of the stock standard solution in ethanol, yielding concentrations of at-RA in the range of 3.25-104 $\mu \mathrm{M}$. After at-RA calibration, samples were diluted by ethanol and sonicated in an ice-water bath for $10 \mathrm{~min}$ prior to injection. The sonication causes the proteinoid NPs to disassemble and elute the RA. The injection volume was set to $50 \mu \mathrm{l}$ for all standard samples in the range of 3.25$104 \mu \mathrm{M}$ at-RA. The weight of drug in each sample was calculated using the calibration curve.

\section{FTIR}

FTIR measurements of the at-RA and proteinoid particles in absence and presence of at-RA were performed by the attenuated total reflectance (ATR) technique, using Bruker ALPHA-FTIR QuickSnap ${ }^{\text {twx }}$ sampling module equipped with Platinum ATR diamond module.

\section{XRD}

All samples were analyzed for the presence of the crystalline and non-crystalline nature by exploiting XRD. The XRD patterns were recorded using an X-ray diffractometer, model D8 Advance (Bruker, Israel) with $\mathrm{Cu}$ Ka radiation. Samples of free at-RA and filled NPs were recorded at an equivalent concentration.

\section{UV-protection of the at-RA by proteinoid NPs}

The test was performed in quartz vials, $5 \mathrm{ml}$ of Prot. 5/RA (20\%) NPs aqueous dispersion $(1 \mathrm{mg} / \mathrm{ml})$ and a control solution of $1 \mathrm{mg}$ at$\mathrm{RA}$ in $5 \mathrm{ml}$ water containing $1 \%$ DMSO were placed in two quartz vials. Both vials were exposed to artificial light in the laboratory and samples were taken at each time period and analyzed by HPLC as described above.

\section{Leakage of at-RA from proteinoid NPs}

The dialysis bag method was used to test whether there is leakage of at-RA from the proteinoid particles [3], into 3 test tubes containing $40 \mathrm{ml}$ water were added 3 dialysis bags filled with $1 \mathrm{ml}$ of Prot. 5/RA NPs dispersion $(1 \mathrm{mg} / \mathrm{ml})$. Each test tube was placed in a different temperature 4,25 and $37^{\circ} \mathrm{C}$, and the water medium was tested for atRA presence by HPLC as described above, at different time points, over a week.

\section{Cell cytotoxicity testing}

Cell cytotoxicity was assessed by measuring the release of LDH into cell culture supernatants. LDH activity was assayed using the Cytotoxicity Detection Kit according to the manufacturer's instructions [32]. HaCaT cells were grown in DMEM supplemented with heatinactivated FBS $10 \%$, penicillin/streptomycin $(100 \mathrm{IU} / \mathrm{ml} / 100 \mu \mathrm{g} / \mathrm{ml})$ and L-glutamine $(2 \mathrm{mM})$. Cells were screened to ensure remained mycoplasma-free using mycoplasma detection kit. HaCaT cells $\left(3 \times 10^{5}\right.$ cells per well) were seeded and grown to $90-95 \%$ confluency in 96 well plates before treatment with the proteinoid particles.

The proteinoid particles were freshly dispersed in water $(1 \mathrm{mg} /$ $\mathrm{ml}$ ) containing $1 \%$ DMSO. Particle dispersion was then added to the 95\% confluent cell culture in culture medium. The cell cultures were further incubated at $37^{\circ} \mathrm{C}$ in a humidified $5 \% \mathrm{CO}_{2}$ incubator and then checked for cellular cytotoxicity after $48 \mathrm{~h}$. Untreated cell cultures were included in all assays as negative control. Cell cultures treated with $1 \%$ Triton-x-100 were used as positive control. The percentage of cell cytotoxicity was calculated using the formula shown in the manufacturer's protocol [32]. All samples were tested in tetraplicates.

\section{Cell permeability}

Prot. 5 and Prot. 5/RA NPs were conjugated to Cy7 and Cy3 in a similar manner. Briefly, following particle preparation, $5 \mathrm{ml}$ of the particle aqueous dispersion $(1 \mathrm{mg} / \mathrm{ml})$ were added to a vial. Then, $10 \mu \mathrm{l}$ of $1 \mathrm{M} \mathrm{NaOH}$ solution were added to the particle dispersion to reach $\mathrm{pH}=8$. The dyes were each dissolved in DMSO to a concentration of $10 \mathrm{mg} / \mathrm{ml}$, and $50 \mu \mathrm{l}$ of the dye solution was then added to the particle dispersion and stirred for $1 \mathrm{~h}$ at $150 \mathrm{rpm}$. The conjugated particle dispersion was dialyzed through a cellulose membrane (1000 Da MWCO) against distilled water for $4 \mathrm{~h}$ to remove the DMSO and excess dye.

FACS of Cy7-conjugated NPs within HaCaT cells was evaluated by FACS Aria III (BD) cell sorter. Cells were treated with Cy7-conjugated Prot. 5 and Prot. 5/RA NPs $(0.1 \mathrm{mg} / \mathrm{ml})$ for $4 \mathrm{~h}$ at $37^{\circ} \mathrm{C}$, prior to analysis. In order to maximize cell viability and minimize mechanical perturbations, the flow rate was set to 1.1 (minimum). For Cy7 analysis $633 \mathrm{~nm}$ excitation laser was used with a filter. Data were processed by FlowJo v7.6.4.

For the Cy3 conjugated particles, HaCaT cells $(50,000)$ were grown on $35 \mathrm{~mm}$ disposable glass-bottomed tissue culture plates (MatTek) for 48 h. Cells were then treated with Cy3-conjugated Prot. 5 and Prot. 5/ RA NPs $(0.1 \mathrm{mg} / \mathrm{ml})$ for $4 \mathrm{~h}$ at $37^{\circ} \mathrm{C}$. After incubation, cells were washed with fresh medium and stained with Hoechst (nucleus staining, $1 \mu \mathrm{g} /$ $\mathrm{ml}$ ) and Alexa Fluor 680 conjugated Wheat germ agglutinin (WGA, cell membrane staining, $1 \mu \mathrm{g} / \mathrm{ml}$ ). Live cell imaging was performed on an Olympus FV-1000 confocal microscope.

\section{Freeze-drying of proteinoid particles for long-term storage}

For long-term storage the particles were freeze-dried, $50 \mathrm{mg}$ of trehalose were added to $5 \mathrm{ml}$ aqueous dispersion of the Prot. 5/RA NPs $(1 \mathrm{mg} / \mathrm{ml})$, followed by lyophilization to dryness. After 3 months storage at $4^{\circ} \mathrm{C}$, the NP powder was redispersed in water to a volume of $1 \mathrm{ml}$ and characterized with respect to particle size, $\zeta$-potential and DL. 
Citation: Belostozky A, Kolitz-Domb M, Grinberg I, Haham H, Margel S (2017) Engineering of New UV-Blocking Hollow Proteinoid Nanoparticles of Narrow Size Distribution Containing All-trans Retinoic Acid for Biomedical Applications. J Nanomed Nanotechnol 8: 462. doi: 10.4172/21577439.1000462

Page 4 of 9

\section{Results and Discussion}

\section{Design and characterization of UV-absorbing proteinoids}

In order to obtain proteinoid NPs that encapsulate and protect at-RA from UV-light, Para-amino benzoic acid (PABA) and three additional natural amino acids, L-glutamic acid (Glu), L-phenylalanine (Phe) and L-tyrosine (Tyr), have been chosen for the synthesis. Glu has to be present in the proteinoid synthesis, since at high temperatures it is condensed into pyroglutamic acid, the initiator and solvent of the polymerization process [33]. Phe and Tyr were incorporated in the proteinoid to provide sufficient mechanical properties (strength and rigidity) due to their aromatic rings content $[24,26]$. PABA, which was widely used in sunscreens (as it absorbs UVB 290-320 nm), is structurally similar to natural amino acids, consisting of a benzene ring substituted with a primary amino group and a carboxylic group in para configuration (Figure 2B) [34]. Therefore, PABA can be integrated into the proteinoid backbone during the thermal step-growth polymerization process. It should also be noted that the aromatic ring of PABA is incorporated in the proteinoid backbone while the aromatic rings of Phe and Tyr are in the side chains of the proteinoid backbone. Therefore, PABA is also used for improving the mechanical properties of the proteinoid shell and as a UV absorber, while the aromatic rings of Phe and Tyr are present in the core of the proteinoid particles to hold the self-assembled structure.

A series of five proteinoids (Prot. 1-5) was prepared using Glu, Phe and Tyr (in 1:1:1 weight ratio), with increasing PABA concentrations $(0,1,5,10$ and 20 wt.\%), as shown in Table 1 .

All proteinoids were characterized for their molecular weights and polydispersity index (PDI) by gel permeation chromatography (GPC) and for their optical activity by a polarimeter. The results are shown in Table 2. The molecular weights of the proteinoids made by the stepgrowth thermal polymerization process were in the range from 42 to

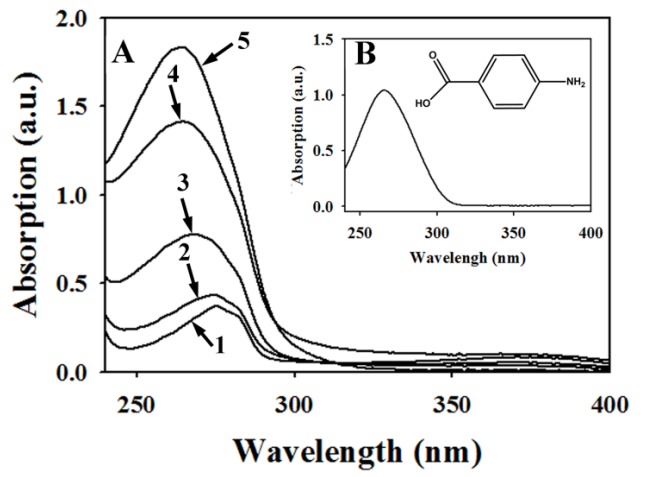

Figure 2: Absorption spectra of the different proteinoids (A) and pure PABA (B). Prot. 1-5 are represented by $1-5$, respectively. The proteinoids and the PABA were scanned for their absorption by spectrophotometer at a concentration of 1 $\mathrm{mg} / \mathrm{ml}$ in $0.1 \mathrm{~N} \mathrm{NaOH}$ aqueous solution.
$84 \mathrm{kDa}$, with low PDIs 1.02-1.12. The presented range of relatively high molecular weights and low PDIs is unexpected for a step-growth polymerization process [28]. Until recently, most of the reported proteinoids in the literature were synthesized from at least seven amino acids and possessed relatively low molecular weights with higher PDIs (above 1.5), as common for step-growth polymerization mechanism [28]. The high molecular weights shown in the present study indicate that the polymerization procedure used here may provide relatively long polymer chains which resemble long proteins [29]. Long polymers usually possess improved mechanical properties than short ones, due to the increased number of physical interactions between the polymer chains [23]. Another important characteristic of the proteinoids is their optical activity, which is reserved after the thermal polymerization process was completed, as shown in Table 2. This fact is important and may be applied in the future for designing stereospecific drug carriers [35].

The proteinoids were scanned for their absorption spectra, in order to study their UV-absorbing ability. The results are shown in Figure 2. The absorption spectra of Prot. 1-5 are illustrated in Figure 2A, while Figure 2B illustrates the absorption spectra of pure PABA. Prot. 1 (1 in Figure 2A), which does not contain PABA, displays a small peak at $278 \mathrm{~nm}$, attributed to Tyr. For prot. 2-5, it is clearly shown that upon increasing the PABA concentration in the proteinoid backbone, the absorbance peak broadens and the intensity increases. Moreover, a slight blue-shift is observed upon increasing the PABA concentrations from 0 to $20 \%$. The shifts in the absorption spectra are caused by the different content of molecules that lead to a difference in the HOMOLUMO interactions [36].

\section{Proteinoid particle formation and optimization}

Hollow and at-RA-filled NPs were prepared from each proteinoid in order to evaluate which proteinoid can produce particles with the best properties in terms of size in diameter, size distribution (SD) and stability in the aqueous continuous phase as measured by $\zeta$-potential. As mentioned previously, proteinoid particles are formed by a selfassembly process. After preparation, the obtained particles were characterized for their size, SD and $\zeta$-potential, as shown in Table 3. Overall, the hollow proteinoid particle diameters were less than $180 \mathrm{~nm}$ with a narrow SD of $8-12 \%$. The narrow SD of the NPs may be attributed to the low PDI of the proteinoid polymers. Prot. 1, consisting of Glu, Phe and Tyr, yielded NPs of $118.2 \pm 14 \mathrm{~nm}$, whereas Prot. 2 which contains $1 \%$ PABA yielded NPs of $180.9 \pm 19 \mathrm{~nm}$. This increase in diameter may be due to the slight increase in the rigidity of the proteinoid backbone caused by the addition 1\% PABA. Table 3 , however, indicates that the addition of PABA above $1 \%$ leads to a decrease in the size of the particles, the size of the proteinoids containing $1,5,10$ and $20 \%$ PABA decreased from $180.9 \pm 19 \mathrm{~nm}$ to $170.3 \pm 13,77.5 \pm 8$ and $56.4 \pm 6 \mathrm{~nm}$, respectively. This decrease in size relative to increasing concentrations of PABA is possibly due to the significant increase of the hydrophobicity of the proteinoid backbone

\begin{tabular}{|c|c|c|c|c|}
\hline Proteinoid & Mw (kDa) & Mn (kDa) & Mp (kDa) & PDI \\
\hline Prot. 1 & 65.0 & 61.9 & 55.3 & 1.05 \\
\hline Prot. 2 & 44.2 & 39.2 & 30.4 & 1.12 \\
\hline Prot. 3 & 42.2 & 39.0 & 33.1 & 1.08 \\
\hline Prot. 4 & 51.7 & 47.5 & 41.6 & 1.08 \\
\hline Prot. 5 & 84.2 & 80.4 & 77.2 & 1.0 \\
\hline
\end{tabular}

Molecular weights were measured by GPC, Mw and $\mathrm{Mn}$ are the weight average and number average molecular weights, respectively, Mp is the molecular weight at the peak; $\mathrm{PDI}$ is the polydispersity index, given by $\mathrm{Mw} / \mathrm{Mn}$; the specific optical rotation was measured at $\mathrm{C}=1$ in $\mathrm{H}_{2} \mathrm{O}, 25^{\circ} \mathrm{C}$.

Table 2: Mw, Mn, Mp, PDI and optical activity of the proteinoids. 
Citation: Belostozky A, Kolitz-Domb M, Grinberg I, Haham H, Margel S (2017) Engineering of New UV-Blocking Hollow Proteinoid Nanoparticles of Narrow Size Distribution Containing All-trans Retinoic Acid for Biomedical Applications. J Nanomed Nanotechnol 8: 462. doi: 10.4172/21577439.1000462

Page 5 of 9

as the PABA concentration increases, leading thereby to shrinking of the hollow proteinoid particles dispersed in the aqueous continuous phase. The RA (5\%)-filled nanoparticles (Prot. 1-5/RA NPs) sizes range from $65.0 \pm 10$ to $179.1 \pm 19 \mathrm{~nm}$, with a narrow SD of $10-12 \%$, displaying an insignificant change in the diameter compared to the hollow corresponding particles. It should be noted that the size of the proteinoid NPs depends on the environment of the self-assembly process, thus upon changing the at-RA or the DMSO concentrations the NPs size changes without a certain predetermined trend.

$\zeta$-Potential values may predict the physical stability of the NPs aqueous dispersions $[37,38]$. The $\zeta$-potential indicates the degree of repulsion between close and similarly charged particles in the aqueous dispersion (negative or positive) and the ability to prevent aggregation of the particles.

Hollow proteinoid particles made from Prot. 1 have a $\zeta$-potential of $-16.9 \pm 2 \mathrm{mV}$ (Table 3 ). The negative charge is derived from the carboxylate groups of the Glu, which reside on the outer part of the particle. Prot. 2 particles have a slightly less negative $\zeta$-potential, -14.5 $\pm 2 \mathrm{mV}$, probably due to the addition of PABA to the proteinoid backbone. Yet, the addition of $20 \%$ PABA to the proteinoid produces the most stable particles, Prot. $5 \mathrm{NPs}$, with $\zeta$-potential of $-19.9 \pm 2 \mathrm{mV}$. These results may be difficult to explain, since the increase in the PABA concentration on the surface of the proteinoid particles on the account of Glu should result in particles of relatively lower $\zeta$-potential. However, these unexpected results may be explained by the fact that the particles containing $20 \%$ PABA possess significantly lower diameter than that prepared in the absence of PABA, $56.4 \pm 6 \mathrm{~nm}$ compared to $118.2 \pm 14$ $\mathrm{nm}$, respectively (Table 3 ).

Table 3 also exhibits, as expected, that the entrapment of at-RA ( $5 \%$ relative to the proteinoid) within the proteinoid particles reduced further their negative $\zeta$-potential, e.g., from $-16.9 \pm 2,-14.5 \pm 2,-15.8$ $\pm 4,-16.3 \pm 3$ and $-19.9 \pm 2 \mathrm{mV}$ for hollow Prot. 1-5 NPs to $-23.6 \pm 2$, $-26.9 \pm 2,-29.7 \pm 3,-36.3 \pm 4$ and $-36.8 \pm 2 \mathrm{mV}$ for Prot. 1-5/RA NPs, respectively. This decrease in the negative charge of the proteinoid NPs due to the entrapment of at-RA can be explained by the addition of carboxylate groups belonging to the at-RA.

The drug loading (DL) capability of proteinoids 1-5 for at-RA was analyzed by high performance liquid chromatography (HPLC), using calibration standard solutions of at-RA in suitable concentrations [31]. Prior to the self-assembly of Prot. 1-5/RA NPs, at-RA was dissolved in DMSO (0.5\% relative to the overall aqueous dispersion). Table 3

\begin{tabular}{|l|c|c|c|}
\hline NPs & Size $\mathbf{( n m})$ & $\begin{array}{c}\zeta \text {-Potential } \\
(\mathbf{m V})\end{array}$ & DL (\%) \\
\hline Prot. 1 & $118.2 \pm 14$ & $-16.9 \pm 2$ & - \\
\hline Prot. 2 & $180.9 \pm 19$ & $-14.5 \pm 2$ & - \\
\hline Prot. 3 & $170.3 \pm 13$ & $-15.8 \pm 4$ & - \\
\hline Prot. 4 & $77.5 \pm 8$ & $-16.3 \pm 3$ & - \\
\hline Prot. 5 & $56.4 \pm 6$ & $-19.9 \pm 2$ & - \\
\hline Prot. 1/RA & $146.7 \pm 14$ & $-23.6 \pm 2$ & $2.6 \pm 0.3$ \\
\hline Prot. 2/RA & $165.5 \pm 19$ & $-26.9 \pm 2$ & $1.2 \pm 0.1$ \\
\hline Prot. 3/RA & $179.1 \pm 19$ & $-29.7 \pm 3$ & $1.4 \pm 0.2$ \\
\hline Prot. 4/RA & $80.5 \pm 9$ & $-36.3 \pm 4$ & $2.4 \pm 0.3$ \\
\hline Prot. 5/RA & $65.0 \pm 10$ & $-36.8 \pm 2$ & $4.0 \pm 0.2$ \\
\hline Proteinoid partin & & \\
\hline
\end{tabular}

Proteinoid particles were made by a self-assembly process in $\mathrm{NaCl} 10^{-5} \mathrm{M}$ aqueous solution containing $0.5 \%$ DMSO. The initial RA concentration was $5 \% \mathrm{w} / \mathrm{w}$ relative to the proteinoid. The proteinoid particles size and SD were measured by DLS, $\zeta$-potential was measured at $\mathrm{pH}=7$ by $\zeta$-potential analyzer and $\mathrm{DL}$ by HPLC, using calibration curve of standard solutions of at-RA.

Table 3: Size, SD, $\zeta$-potential and DL of the proteinoid NPS.

\begin{tabular}{|c|c|c|c|}
\hline$\%$ at-RA & Size (nm) & $\zeta$-Potential (mV) & DL (\%) \\
\hline 1 & $71.0 \pm 8$ & $-23.6 \pm 1.5$ & $1 \pm 0.1$ \\
\hline 5 & $97.2 \pm 10$ & $-25.1 \pm 2$ & $5 \pm 0.1$ \\
\hline 10 & $66.9 \pm 8$ & $-26.9 \pm 2$ & $10 \pm 0.1$ \\
\hline 20 & $85.5 \pm 10$ & $-30.3 \pm 3$ & $20 \pm 0.1$ \\
\hline 30 & $94.8 \pm 10$ & $-30.3 \pm 3$ & $20 \pm 0.3$ \\
\hline
\end{tabular}

Proteinoid particles were made by self-assembly in $10^{-5} \mathrm{M} \mathrm{NaCl}$ solution containing $1 \%$ DMSO. The proteinoid particles size and SD were measured by DLS, $\zeta$-potential was measured at $\mathrm{pH}=7$ by $\zeta$-potential analyzer and $\mathrm{DL}$ was measured by HPLC, using calibration with standard solutions of at-RA.

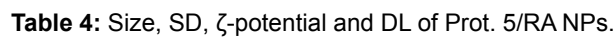

indicates that from the 5\% at-RA used for the encapsulation process, the DL\% of Prot. 1-5/RA NPs was $2.6 \pm 0.3,1.2 \pm 0.1,1.4 \pm 0.2,2.4 \pm 0.3$ and $4.0 \pm 0.2 \%$, respectively.

As shown in the table, Prot. 5 showed the most promising properties for particles encapsulating at-RA. Hollow particles made from Prot. 5 were the smallest, with a diameter of $56.4 \pm 6 \mathrm{~nm}$ and had the best dispersion stability of $-19.9 \pm 2 \mathrm{mV}$. Furthermore, RA-filled particles, Prot. 5/RA NPs, had the highest DL of $4 \pm 0.2 \%$ (out of the maximum $5 \%)$ and the best stability value of $-36.8 \pm 0.2 \mathrm{mV}$, with NPs size of 65.0 $\pm 10 \mathrm{~nm}$. For the PABA-containing proteinoid NPs, the stability and the DL raise as the PABA percentage raises in the proteinoid backbone, probably due to strong hydrophobic interactions between PABA and at-RA.

In order to optimize the DL, different at-RA concentrations $(1,5$, 10, 20 and 30\%) were encapsulated within Prot. 5 particles, as shown in Table 4. The DMSO concentration was increased from $0.5 \%$ to $1 \%$ relative to the overall aqueous dispersion, in order to increase the solubility of the at-RA in the aqueous continuous phase. These trials indicated that the maximal DL capacity was $20 \%$ of at-RA in Prot. 5 particles. Raising the at-RA percentage above $20 \%$ did not affect the encapsulation efficiency at all. Our measurements also indicated that Prot. 5/RA (20\%) NPs possess a diameter of $85.5 \pm 10 \mathrm{~nm}$ and $\zeta$-potential of $-30.3 \pm 3 \mathrm{mV}$. It should be noted that the DMSO was removed by dialysis and no solid free RA was observed in the reaction vials, indicating that the encapsulation was successful. Due to these results, Prot. 5 and Prot. 5/RA (20\%) NPs were chosen as optimal for further investigation.

\section{Characterization of Prot. 5 and Prot. 5/RA (20\%) NPs}

Hollow Prot. 5 NPs and Prot. 5/RA (20\%) NPs were characterized by dynamic light scattering (DLS) and by cryogenic transmission electron microscopy (cryo-TEM), as shown in Figure 3.

Prot. 5 produced a single population of hollow spherical particles of $56 \pm 6 \mathrm{~nm}$ diameter as shown by the DLS histogram (Figure $3 \mathrm{~A}$ ) and $50 \pm 15 \mathrm{~nm}$ as shown by the cryo-TEM measurements (Figure $3 \mathrm{~B}$ ).

RA-filled particles, Prot. 5/RA (20\%) NPs, were larger, containing a single population with a diameter of $85 \pm 10 \mathrm{~nm}$ according to the DLS measurements (Figure $3 \mathrm{C}$ ) and $82 \pm 13 \mathrm{~nm}$ as measured by the cryoTEM (Figure 3D).

It is well-known that such nanometric size particles are able to penetrate the skin $[15,16]$. Skin penetration is an important advantage of nano-scaled particles, since the drug (at-RA) would be able to effect deeper layers of the skin, resulting in a better therapeutic effect on the skin.

To further characterize the optimal proteinoid particles Fourier transform infrared spectroscopy (FTIR) measurements and X-ray 
Citation: Belostozky A, Kolitz-Domb M, Grinberg I, Haham H, Margel S (2017) Engineering of New UV-Blocking Hollow Proteinoid Nanoparticles of Narrow Size Distribution Containing All-trans Retinoic Acid for Biomedical Applications. J Nanomed Nanotechnol 8: 462. doi: 10.4172/21577439.1000462

Page 6 of 9

powder diffraction (XRD) analysis were performed on at-RA powder, Prot. 5 NPs and Prot. 5/RA (20\%) NPs (Figure 4).

The at-RA powder (Figure $4 \mathrm{~A}$ ) demonstrated characteristic peaks of alkenes: $=\mathrm{C}-\mathrm{H}$ stretch at $959 \mathrm{~cm}^{-1}, \mathrm{C}=\mathrm{C}$ stretch at $1680 \mathrm{~cm}^{-1}$ and $\mathrm{C}-\mathrm{H}$ stretch at $2931 \mathrm{~cm}^{-1}$. Furthermore, characteristic peaks of carboxylic acid were shown: $\mathrm{O}-\mathrm{H}$ band at $913 \mathrm{~cm}^{-1}$ and $\mathrm{C}-\mathrm{O}$ stretch at $1181 \mathrm{~cm}$ ${ }^{1}$. Freeze-dried Prot. 5 hollow NPs (Figure 4B) showed characteristic peaks of proteinoids: C-N stretch at $1022 \mathrm{~cm}^{-1}$ and at $1250 \mathrm{~cm}^{-1}$ of amine, $\mathrm{C}=\mathrm{O}$ stretch at $1674 \mathrm{~cm}^{-1}$ of ketone and N-H stretch at 3243 $\mathrm{cm}^{-1}$ of amide [28]. Freeze-dried Prot. 5/RA NPs (Figure 4C) FTIR
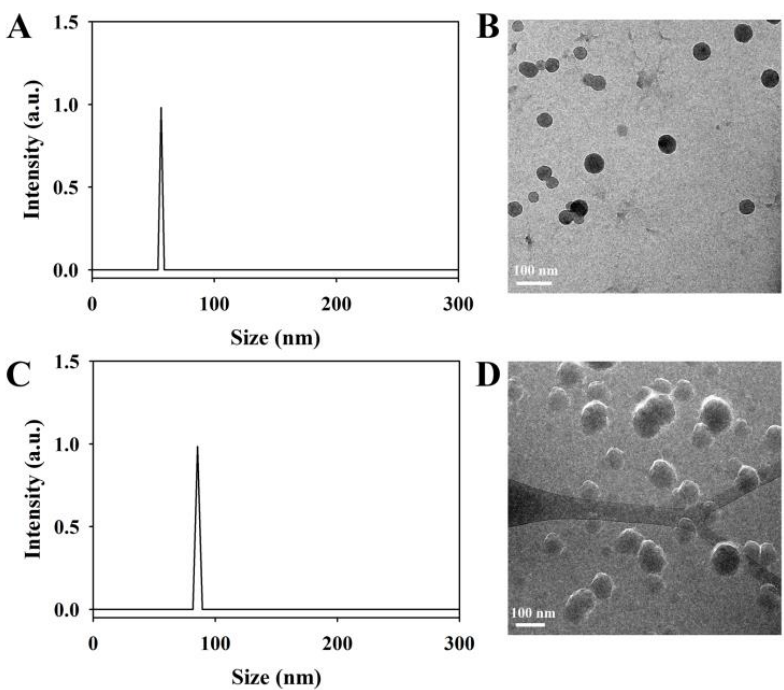

Figure 3: Hydrodynamic size histograms and Cryo-TEM images of Prot. 5 NPs $(A$ and $B)$ and Prot. 5/RA (20\%) NPs (C and D).

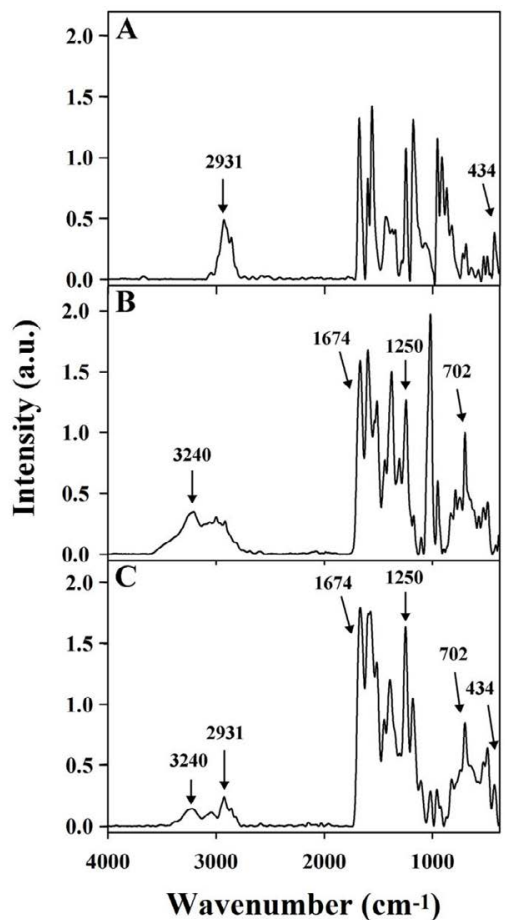

spectrum appears to be a combination of peaks from the Prot. 5 and the at-RA. For instance, common peaks for Prot. 5 and Prot. 5/RA NPs appear at the fingerprint area $494 \mathrm{~cm}^{-1}$ and $702 \mathrm{~cm}^{-1}$, amine stretch at $1250 \mathrm{~cm}^{-1}$, ketone $\mathrm{C}=\mathrm{O}$ stretch at $1674 \mathrm{~cm}^{-1}$ and amide $\mathrm{N}-\mathrm{H}$ stretch at $3240 \mathrm{~cm}^{-1}$. Common peaks for at-RA and Prot. 5/RA NPs appear also at the fingerprint area $432 \mathrm{~cm}^{-1}$ and an alkene C-H stretch at $2931 \mathrm{~cm}^{-1}$. These results suggest that the RA was successfully encapsulated within the proteinoid NPs.

XRD analysis of at-RA powder and freeze-dried Prot. 5 and Prot. 5/RA (20\%) NPs are shown in Figure 4D-4F. The diffraction pattern of at-RA shows characteristic sharp peaks of crystalline at-RA (Figure 4D), whereas the Prot. 5 NPs show a typical amorphous pattern, as expected for a random polymerization product (Figure 4E). Crystalline at-RA peaks are not detected in the diffractogram of the freeze-dried Prot. 5/RA NPs (Figure 4F), indicating that the proteinoid stabilizes the encapsulated RA in an amorphous form. In a case where the RA was located on the outside of the particles, crystallization of the RA would occur, which should have affected the diffraction patterns of Prot. 5/ RA NPs. This suggests that the drug was successfully encapsulated within the proteinoid particles. Moreover, it seems that the at-RA did not change the nature of the proteinoid particles, also attesting the encapsulation of at-RA.

\section{UV-protection of at-RA by the proteinoid NPs}

As mentioned previously, at-RA is known to be unstable in aqueous solutions and degrades easily when exposed to both sunlight and artificial light [14]. To examine the protection ability of the proteinoid NPs, free at-RA and Prot. 5/RA NPs dispersed in aqueous based continuous phase, were exposed to artificial light for $24 \mathrm{~h}$. The results are shown in Figure 5. The RA-filled particle dispersion and the free at-RA solution were placed in separate quartz vials and exposed to artificial light over $24 \mathrm{~h}$ simultaneously. Samples were taken at various

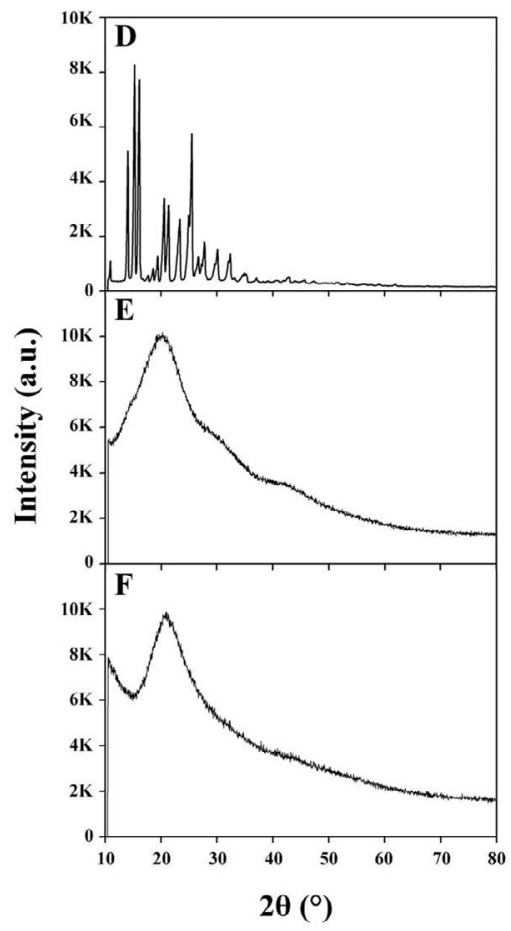

Figure 4: FTIR spectra and XRD diffraction patterns of at-RA powder (A and D), Prot. 5 NPs (B and E) and Prot. 5/RA (20\%) NPs (C and F) respectively. 
Citation: Belostozky A, Kolitz-Domb M, Grinberg I, Haham H, Margel S (2017) Engineering of New UV-Blocking Hollow Proteinoid Nanoparticles of Narrow Size Distribution Containing All-trans Retinoic Acid for Biomedical Applications. J Nanomed Nanotechnol 8: 462. doi: 10.4172/21577439.1000462

Page 7 of 9

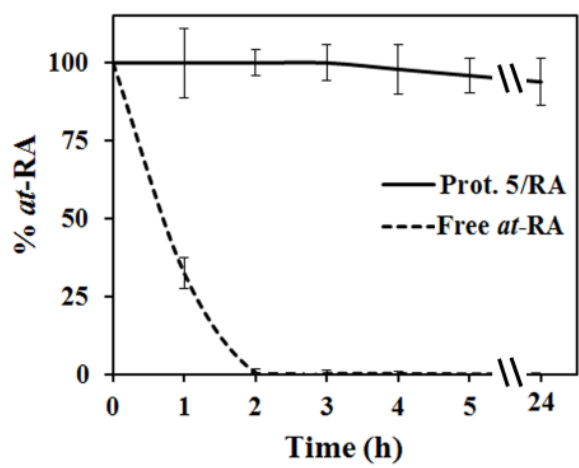

Figure 5: Degradation of at-RA after exposure of free at-RA and encapsulated in Prot. 5 NPs to artificial light. Prot. 5/RA (20\%) NPs were prepared according to the methods section, \% at-RA was calculated by HPLC, using calibration curve of standard solutions of at-RA.

time points and the concentration of at-RA was measured by HPLC. The free at-RA degraded entirely over $3 \mathrm{~h}$ under the experimental conditions, while Prot. 5/RA NPs were able to protect the drug from degradation up to $94 \%$ over $24 \mathrm{~h}$. The proteinoid particles, which contain UV-absorbing molecules Tyr and PABA in the proteinoid backbone, protect the at-RA from direct light. Moreover, the proteinoid particles can keep the at-RA stable in an aqueous dispersion while no leakage was detected from the NPs to the aqueous medium. Therefore, the proteinoid NPs can stabilize the encapsulated at-RA significantly over free at-RA.

\section{In-vitro cell cytotoxicity study of the proteinoid NPs}

$\mathrm{HaCaT}$ cell line was used in the in-vitro experiments as a model for topical treatment [39]. HaCaT is a spontaneously transformed human epithelial cell line from adult skin. It is the first permanent epithelial cell line that exhibits normal differentiation and provides a useful tool for studying regulation of keratinization in human cells [40].

Cell cytotoxicity of the proteinoid particles was assessed by measuring the release of cytoplasmic lactate dehydrogenase (LDH) into the cell culture supernatants [32]. When tested by the LDH quantitative assay, hollow Prot. 5 and Prot. 5/RA (20\%) NPs aqueous dispersions $(0.1$ and $0.01 \mathrm{mg} / \mathrm{ml})$ had no cytotoxic effect on the HaCaT cell line compared to untreated cells, as demonstrated in Figure 6. Hence, hollow Prot. 5 and Prot. 5/RA NPs can be suitable candidates for topical treatment, considering their non-toxicity to epithelial cells.

\section{In-vitro cell permeability study of the proteinoid NPs}

The cell permeability of the proteinoid particles was analyzed by flow cytometry (FACS) and visualized by a confocal microscope. The results are shown in Figure 7. The proteinoid particles (hollow Prot. 5 and Prot. 5/RA NPs) were conjugated, as described in the methods section, to the fluorescent dyes Cy7 and Cy3 for FACS and confocal microscope observation, respectively. Figure 7A demonstrates efficient cell permeability of the Cy7-conjugated Prot. 5 and Prot. 5/RA NPs to the $\mathrm{HaCaT}$ cells post incubation of $4 \mathrm{~h}$ at $37^{\circ} \mathrm{C}$ prepared as described in the methods section. The Cy3-conjugated Prot. 5 and Prot. 5/RA NPs were incubated with $\mathrm{HaCaT}$ cells in the same manner for microscope visualization. Prior to visualization, cells were stained with Hoechst 33342 and Alexa-red conjugated Wheat Germ Agglutinin (WGA) for nucleus and cell membrane, respectively [41,42]. Cells were then observed by a confocal microscope as shown in Figures 7B and 7C. It is clearly observed that Prot. 5 and Prot. 5/RA NPs have sufficient cell

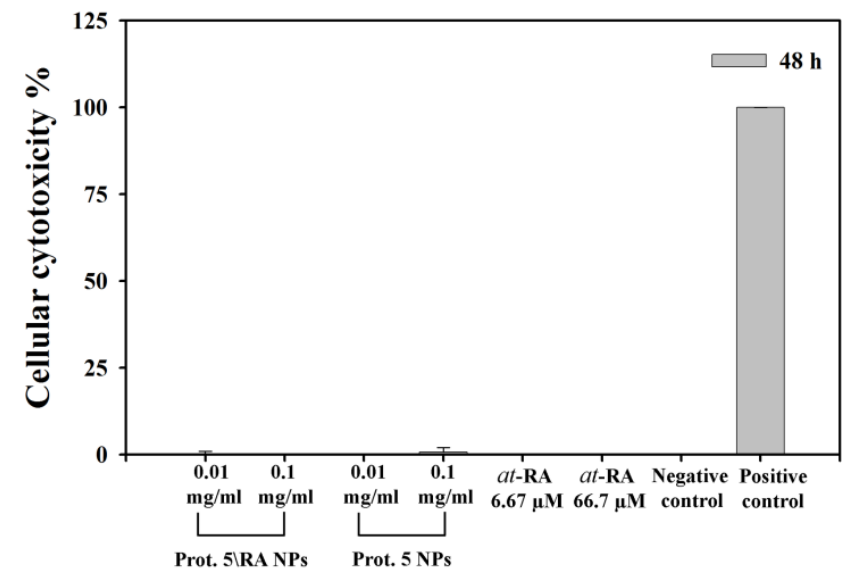

Figure 6: Cytotoxic effect of the RA-filled and hollow Prot. 5 NPs on HaCaT cells measured by the LDH assay. HaCaT cells were seeded onto a 96 well plate $\left(3 \times 10^{5} /\right.$ well $)$ and were grown to $95 \%$ confluence. Then, cells were incubated for $48 \mathrm{~h}$ with the NPs dispersed in water containing 1\% DMSO $(0.01$ and $0.1 \mathrm{mg} / \mathrm{ml}$ ) and relative amounts of free RA. Cells were incubated with Triton-x-100 $1 \%$ as positive control. Negative control samples (untreated cells) were similarly incubated with water containing $1 \%$ DMSO. Each bar represents mean \pm standard deviation of 4 separate samples.

\begin{tabular}{|c|c|c|c|}
\hline Prot. 5/RA NP dispersion & Size $(\mathrm{nm})$ & 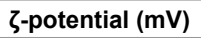 & DL (\%) \\
\hline Fresh & $85.45 \pm 10$ & $-30.3 \pm 3$ & $20 \pm 0.1$ \\
\hline Freeze-dried & $84.4 \pm 10$ & $-30.3 \pm 3$ & $20 \pm 0.2$ \\
\hline
\end{tabular}

Measurements were performed on Prot. 5/RA NPs aqueous dispersions of freshly prepared and after freeze-drying and redispersion. The proteinoid particle size and SD were measured by DLS, $\zeta$-potential was measured at $\mathrm{pH}=7$ by $\zeta$-potential analyzer and DL was measured by HPLC, using calibration curve of standard solutions of at-RA.

Table 5: Size, SD, $\zeta$-potential and DL of the Prot. 5/RA NPs.

penetration after $4 \mathrm{~h}$ of incubation. These results can be explained by the fact that the proteinoid NPs are similar to proteins, hence the cells may utilize them as nutrients [29]. Since, the at-RA is protected by the proteinoid particles, higher concentration of at-RA can penetrate the cells in comparison to free at-RA, since free at-RA can be converted to a less potent form, from the time it is applied until the time it reaches the cell DNA. Moreover, there is a high concentration of at-RA in each particle, so a higher amount of at-RA will penetrate and affect the cells.

\section{Storage stability of the proteinoid particles}

For long-term storage, the proteinoid NPs containing at-RA were freeze-dried with $1 \%(\mathrm{w} / \mathrm{v})$ trehalose, a natural alpha-linked disaccharide. Trehalose is known to prevent denaturation and aggregation of proteins, while preserving their natural form [43]. Without the addition of trehalose Prot. 5/RA NPs cannot be freezedried and then redispersed. After the NPs were lyophilized to dryness and redispersed in an aqueous phase the particles size, $\zeta$-potential and DL were not affected, as shown in Table 5. Moreover, the NPs can be redispersed to a higher concentration $(5 \mathrm{mg} / \mathrm{ml})$ than the initial one, without any effect on size, $\zeta$-potential or DL. Trehalose is non-toxic and can be easily removed by dialysis. This indicates that the Prot. 5/ RA NPs may be stored and handled as a freeze-dried powder and redispersed upon use.

\section{Conclusions}

In this study, a series of proteinoids containing Glu, Phe, Tyr and PABA was synthesized to form proteinoid NPs that encapsulate photo- 
Citation: Belostozky A, Kolitz-Domb M, Grinberg I, Haham H, Margel S (2017) Engineering of New UV-Blocking Hollow Proteinoid Nanoparticles of Narrow Size Distribution Containing All-trans Retinoic Acid for Biomedical Applications. J Nanomed Nanotechnol 8: 462. doi: 10.4172/21577439.1000462

Page 8 of 9
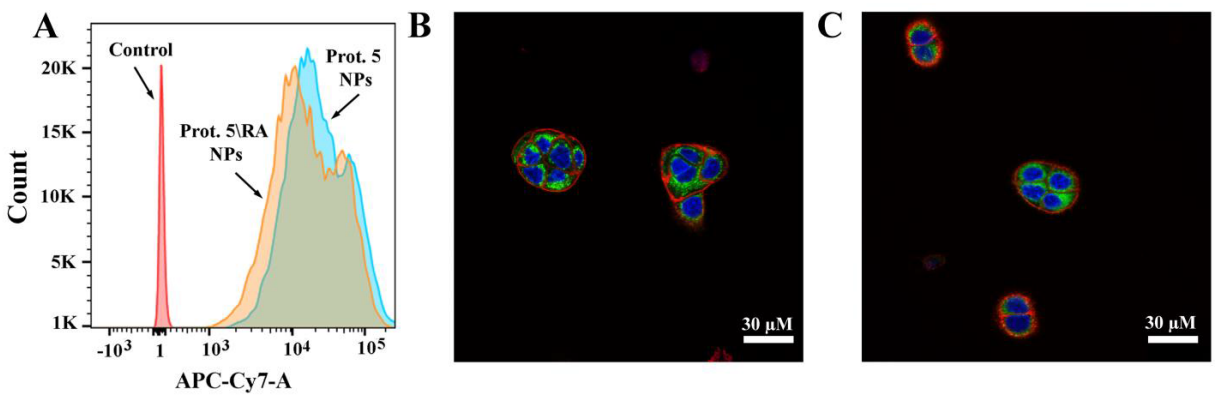

Figure 7: Cell permeability of the proteinoid particles: FACS analysis of Cy7-conjugated Prot. 5 and Prot. 5/RA NPs (A), intracellular accumulation of Cy3-conjugated Prot. 5 NPs (B) and Prot. 5/RA NPs (C) by HaCaT cells, represented by green. Confocal image of live HaCaT cells were stained with Alexa red WGA (cell membrane, red) and Hoechst dye (cell nucleus, blue), visualized with an Olympus FV-1000 confocal microscope, while each dye was sequentially excited, images B and C are merged versions of the microscope images.

sensitive at-RA for topical treatment of acne vulgaris and potentially other types of skin diseases. The proteinoid polymers, synthesized by thermal step-growth polymerization, were mostly uniform in size distribution with relatively high molecular weights. Following proteinoids synthesis, NPs were prepared by a self-assembly procedure and characterized for their size, SD and $\zeta$-potential. Hollow Prot. 5 NPs formed the most stable particle dispersion and the smallest NPs in terms of diameter. Then, the proteinoids were examined for their at-RA encapsulation ability, using HPLC analysis. Again, Prot. 5 had the best characteristics and was able to encapsulate the highest amount of at-RA and form stable, nano-sized particles. After an optimization of the RA-filled particles, Prot. 5/RA (20\%) NPs, as well as hollow Prot. 5 NPs were further characterized by cryo-TEM, FTIR and XRD. The measurements confirmed that at-RA was successfully encapsulated within the proteinoid particles and formed uniform nano-scaled particles. Such particles can be candidates for dermatologic treatment, as similar particles are known to be skin-permeable. Most importantly, it was shown that the proteinoid particles protect at-RA from lightinduced degradation, as $94 \%$ of the drug remained stable over $24 \mathrm{~h}$ of exposure to artificial light, while under similar conditions free alltrans retinoic acid degraded completely over $3 \mathrm{~h}$. The proteinoid NPs are non-toxic to human epithelial cells and were shown to be cellpermeable after $4 \mathrm{~h}$ of incubation under the experimental conditions. For long-term storage the particles were freeze-dried in the presence of $1 \%$ trehalose $(\mathrm{w} / \mathrm{v})$ and redispersed successfully. Hence, proteinoid particles encapsulating at-RA may potentially become useful as a drug delivery system for topical treatment of different skin conditions. Our future plans include assessment of the protected at-RA for use in other biomedical applications such as cancer and auto-immune skin diseases, following suitable adjustments and optimization.

\section{References}

1. Rolewski SL (2003) Clinical review: topical retinoids. Dermatol Nurs 15: 1-16.

2. Serri R, lorizzo M (2008) Cosmeceuticals: focus on topical retinoids in photoaging. Clin Dermatol 26: 633-635.

3. Araseki M, Yamamoto K, Miyashita K (2016) Oxidative stability of polyunsaturated fatty acid in phosphatidylcholine liposomes oxidative stability of polyunsaturated fatty acid. Biosci Biotechnol Biochem 8451: 2573-2577.

4. Nakatsuji T, Kao MC, Fang JY, Zouboulis CC, Zhang L, et al. (2009) Antimicrobial property of lauric acid against Propionibacterium acnes: its therapeutic potential for inflammatory acne vulgaris. J Invest Dermatol 129: 2480-2488.

5. Date A, Naik B, Nagarsenker MS (2006) Novel drug delivery systems: potential in improving topical delivery of antiacne agents. Skin Pharmacol Physiol 19: 2-16.

6. Ourique A, Melero A, Silva CDBD, Schaefer UF, Pohlmann AR, et al. (2011)
Improved photostability and reduced skin permeation of tretinoin: Development of a semisolid nanomedicine. Eur J Pharm Biopharm 79: 95-101.

7. Bergstrom KG (2009) Beyond tretinoin: cosmeceuticals for aging skin. J Drugs Dermatol 8: 674-677

8. Fu JJJ, Hillebrand GG, Raleigh P, Li J, Marmor MJ, et al. (2010) A randomized controlled comparative study of the wrinkle reduction benefits of a cosmetic niacinamide/peptide/retinyl propionate product regimen vs. a prescription $0.02 \%$ tretinoin product regimen. Br J Dermatol. 162: 647-654.

9. Elbuluk OAON (2017) Melasma: An Up-to-Date Comprehensive Review. Dermatol Ther 3: 305-318.

10. Model G (2017) In cutaneous leishmaniasis, induction of retinoic acid in skinderived Langerhans cells is not sufficient for induction of parasite persistencemediating regulatory T cells. J Dermatol Sci 3: 307-309.

11. Wang X, Chen X, Li J, Zhang H, Liu J, et al. (2017) ScienceDirect MiR-200a expression in CD4+ $\mathrm{T}$ cells correlates with the expression of Th17/Treg cells and relevant cytokines in psoriasis vulgaris: A case control study. Biomed Pharmacother 93: 1158-1164

12. Tang X, Gudas LJ (2011) Retinoids, Retinoic Acid Receptors, and Cancer. Annu Rev Pathol Mech Dis 6: 345-64.

13. Venkatesh K, Srikanth L, Vengamma B, Chandrasekhar C, Sanjeevkumar A, et al. (2013) In vitro differentiation of cultured human CD34+ cells into astrocytes. Neurol India 61: 383-388.

14. Curley RW, Fowble JW (1988) Photoisomerization of retinoic acid and its photoprotection in physiologic-like solutions. Photochem Photobiol 47: 831 835 .

15. Gupta A, Singh S, Kotla NG, Webster TJ (2015) Formulation and evaluation of a topical niosomal gel containing a combination of benzoyl peroxide and tretinoin for antiacne activity. Int J Nanomedicine 10: 171-182.

16. Escobar-Chávez JJ, Domínguez-Delgado CL, Díaz-Torres R, Domínguez CL, Ángeles-Anguiano E (2012) Nanocarriers for transdermal drug delivery. Eur Pharm Biopharm 77: 1-2.

17. Fox SW (1960) How did life begin? Science 132: 200-208.

18. Fox SW (1974) The proteinoid theory of the origin of life and competing ideas Am Biol Teach 36: 161-172

19. Fox SW (1995) Thermal synthesis of amino acids and the origin of life. Geochim Cosmochim Acta 59: 1213-1214.

20. Fox SW, Jungck JR, Nakashima T (1974) From proteinoid microsphere to contemporary cell: formation of internucleotide and peptide bonds by proteinoid particles. Orig Life 5: 227-237.

21. Fox SW, Mccauley RJ, Fukushim.T, Windsor CR (1967) Selective action in boundaries of particles of thermal proteinoid. Fed Proc 26: 749.

22. Fox SW, Nakashima T, Przybylski A (1982) The updated experimental proteinoid model. Int. J Nanomedicine 22: 195-204.

23. Shikanov A, Kumar N, Domb AJ (2005) Biodegradable polymers: An update. Isr J Chem 45: 393-399. 
Citation: Belostozky A, Kolitz-Domb M, Grinberg I, Haham H, Margel S (2017) Engineering of New UV-Blocking Hollow Proteinoid Nanoparticles of Narrow Size Distribution Containing All-trans Retinoic Acid for Biomedical Applications. J Nanomed Nanotechnol 8: 462. doi: 10.4172/21577439.1000462

Page 9 of 9

24. Kiel S, Kolitz-Domb M, Corem-Salkmon E, Grinberg I, Margel S (2017) Engineered doxorubicin delivery system using proteinoid-poly ( L-lactic acid) polymeric nanoparticles of narrow size distribution and high molecular weight for cancer treatment. Int J Nanotech Nanomed 2: 1-11.

25. Urry D, Peng S (1995) Nonlinear mechanical force induced pKa shifts: implications for efficiency of conversion to chemical energy. Am Chem Soc 117: 8478-8479.

26. Madhan Kumar A, Panduranga Rao K (1998) Preparation and characterization of $\mathrm{pH}$-sensitive proteinoid microspheres for the oral delivery of methotrexate. Biomaterials 19: 725-732

27. Quirk S (2013) Enhanced catalytic activity from proteinoid microspheres. J Biomed Mater Res Part A 101: 1133-1143.

28. Kolitz-Domb M, Grinberg I, Corem-Salkmon E, Margel S (2014) Engineering of near infrared fluorescent proteinoid-poly (L-lactic acid) particles for in vivo colon cancer detection. J Nanobiotechnology 12 30-43.

29. Kolitz-Domb M, Corem-Salkmon E, Grinberg I, Margel S (2014) Synthesis and characterization of bioactive conjugated near-infrared fluorescent proteinoidpoly(L-lactic acid) hollow nanoparticles for optical detection of colon cancer. Int J Nanomedicine 9: 5041-5053.

30. Kolitz-Domb M, Margel S (2014) Engineered narrow size distribution high molecular weight proteinoids, proteinoid-poly(L-lactic acid) copolymers and nano/micro-hollow particles for biomedical applications. J Nanomed Nanotechnol 5: 1-10

31. Das S, Ng WK, Kanaujia P, Kim S, Tan RBH (2011) Formulation design, preparation and physicochemical characterizations of solid lipid nanoparticles containing a hydrophobic drug: Effects of process variables. Colloids Surf B 88: 483-489.

32. Decker T, Lohmann-Matthes ML (1988) A quick and simple method for the quantitation of lactate dehydrogenase release in measurements of cellula cytotoxicity and tumor necrosis factor (TNF) activity. J Immunol Methods 115: 61-69.
33. Harada K, Fox SW (1958) The thermal condensation of glutamic acid and glycine to linear peptides. J Am Chem Soc 80: 2694-2697.

34. Yoshino K, Takao T, Murata H, Shimonishi Y (1995) Use of the derivatizing agent 4-aminobenzoic acid 2-(diethylamino)ethyl ester for high-sensitivity detection of oligosaccharides by electrospray ionization mass spectrometry. Anal Chem 67: 4028-31.

35. Slager J, Domb AJ (2003) Biopolymer stereocomplexes. Adv Drug Deliv Rev 55: 549-583.

36. Malmström BG (1992) Chemistry 1981-1990.World Scientific

37. Das S, Chaudhury A (2011) Recent advances in lipid nanoparticle formulations with solid matrix for oral drug delivery. AAPS PharmSciTech 12: 62-76.

38. Freitas C, Müller RH (1998) Effect of light and temperature on zeta potentia and physical stability in solid lipid nanoparticle (SLN®) dispersions. Int J Pharm 168: $221-229$.

39. Ridolfi DM, Marcato PD, Justo GZ, Cordi L, Machado D, et al. (2012) Chitosansolid lipid nanoparticles as carriers for topical delivery of tretinoin. Colloids Surf B 93: $36-40$

40. Boukamp P (1988) Normal keratinization in a spontaneously immortalized. J Cell Biol 106: 761-771

41. Juergens A (1975) Recent developments in the detection of deoxyribonucleic acid synthesis by 33258 Hoechst fluorescence. J Histochem Cytochem 23 . 493-505.

42. Voloshina NP, Haugland RP, Stewart JB, Bhalgat MK, Millard PJ, et al. (1999) Alexa dyes, a series of new fluorescent dyes that yield exceptionally bright, photostable conjugates. J Histochem Cytochem 47: 1179-1188.

43. Jain NK, Roy I (2009) Effect of trehalose on protein structure. Protein Sci 18 : 24-36. 